\title{
Mehrfach ungesättigte Fettsäuren PUFA: Ein wichtiger Bestandteil in Zellstoffwechsel und Ernährung
}

\author{
Reinhard Saller¹, Christine Römer-Lüthi², Reto Brignoli3, Remy Meier ${ }^{4}$ \\ 1 UniversitätsSpital, Institut für Naturheilkunde, CH-Zürich; ${ }^{2}$ Ausbildungszentrum Insel, $\mathrm{CH}-\mathrm{Bern}$; ${ }^{3}$ Tradyser $\mathrm{GmbH}$, CH-Rüschlikon; \\ 4Medizinische Universitätsklinik, Abteilung für Gastroenterologie, CH-Liestal
}

$\mathrm{M}$ ehrfach ungesättigte Fettsäuren (Polyunsaturated Fatty Acids = PUFA) sind ein wichtiger Bestandteil praktisch aller Lipide. Unter Lipiden versteht man im Allgemeinen Triglyzeride; diese dienen im Fettdepot der Fettgewebszellen als Energiespeicher. Zellmembranen sind zum grössten Teil aus Phospholipiden aufgebaut und enthalten keine Triglyzeride. PUFA sind sowohl Bestandteil des zellulären Fettdepots als auch wichtige Bausteine der Zellmembranlipide.

\section{Physiologie und biologische Wirksamkeit}

PUFA haben mehr als eine Doppelbindung und werden unter anderem aufgrund der Lokalisation der letzten Doppelbindung in der Kohlenstoffkette klassifiziert. Demnach liegt bei Omega3-Fettsäuren (n-3-Fettsäuren), vom Kettenende her gezählt, die erste Doppelbindung am dritten Kohlenstoffatom, bei Omega-6-Fettsäuren (n-6Fettsäuren) entsprechend am sechsten Kohlenstoffatom (Abbildung 1).

Zwei mehrfach ungesättigte Fettsäuren, die Linolsäure (n-6-PUFA) und die Alpha-Linolensäure (n-3-PUFA) sind für den Menschen essenziell, da ihre charakteristischen Doppelbindungen durch die menschlichen Enzymsysteme nicht eingeführt werden können. Linolsäure (LA) und Alpha-Linolensäure (ALA) müssen demzufolge mit der Nahrung zugeführt werden. Im Gegensatz dazu können die Doppelbindungen von Ölsäure (n-9-Fettsäure) und den längerkettigen Derivaten von Ölsäure vom Menschen aufgebaut werden.
Hintergrund: Mehrfach ungesättigte Fettsäuren (Polyunsaturated Fatty Acids = PUFA) sind ein wichtiger Bestandteil praktisch aller Lipide und damit Bestandteile des zellulären Fettdepots sowie Bausteine der Zellmembranen. Zielsetzung: Erstellung eines Positionspapiers für PUFA. Methoden: Systematische Analyse und Bewertung von Humanstudien (prospektive Doppelblindstudien, epidemiologische und retrospektive Studien, kurzfristige biochemische/hämatologische Studien («Surrogate Markers»)) der letzten 10 Jahre aus den gängigen elektronischen Datenbanken sowie der Angaben von Standardwerken und publizierten Monographien. Ergebnisse und Schlussfolgerungen: Zwei mehrfach ungesättigte Fettsäuren, die Linolsäure und die Alpha-Linolensäure sind für den Menschen essenziell. Die diversen PUFA sind in verschiedenen Ölen enthalten, vor allem in Fischöl. n-3-PUFA aus Fischöl müssen mit Antioxidantien kombiniert werden, damit sie stabilisiert werden und ihre Wirkung entfalten können. Die Wahrscheinlichkeit einer unzureichenden Versorgung mit PUFA ist am grössten bei Menschen, deren Bedarf erhöht ist, zum Beispiel bei Infektionen, chronischen Krankheiten oder in Wachstumsphasen. Im Sinne einer präventiv wirksamen Zusammensetzung der mehrfach ungesättigten Fettsäuren in der Nahrung sprechen sich deutsche, österreichische und schweizerische Ernährungsgesellschaften dafür aus, dass das Verhältnis von Alpha-Linolensäure (n-3) zu Linolsäure (n-6) auf mindestens 1:5 erhöht wird. PUFA haben auf verschiedene Organsysteme und bei verschiedenen Erkrankungen eine positive Wirkung: Hautkrankheiten, koronare Herzkrankheit, obstruktive Lungenleiden und rezidivierende Infekte, senkt die postoperative infektiöse Morbidität. Alpha-Linolensäure bietet praktische Vorteile gegenüber Fischölpräparaten: Kein unangenehmes Aufstossen für den Patienten und geringerer Bedarf an Stabilisatoren (Antioxidantien) in der galenischen Darreichungsform.

Schlüsselwörter: Mehrfach ungesättigte Fettsäuren, Stoffwechsel, Ernährung, Fischöl

\section{Polyunsaturated Fatty Acids (PUFAs): Essential Component of Cellular Metabolism and Nutrition}

Background: Polyunsaturated fatty acids (PUFAs) are an important component of practically all lipids and are therefore components of cellular fat stores and the structural building blocks of the cell membrane. Objective: To draw up a position paper for PUFA. Methods: Systematic analysis and rating of human studies (prospective double-blind studies, epidemiologic and retrospective studies, short period biochemical/haematological studies ("surrogate markers")) of the last 10 years from major data bases, coupled to information in standard works and published monographs. Results and Conclusions: Two polyunsaturated fatty acids, linoleic acid and alpha-linolenic acid, are essential for man. The various PUFAs are contained in various oils, particularly fish oils. n-3 PUFAs from fish oils must be combined with antioxidants, to stabilise them and to maintain their activity. The probability of inadequate PUFA supply is greatest in people with raised requirements, for example, during infections, chronic disease or in phases of growth. German, Austrian and Swiss nutritional societies have expressed the opinion that the ratio of alpha-linolenic acid $(n-3)$ to linoleic acid (n-6) should be raised to at least 1:5, to optimise preventive activity. PUFAs have favourable effects on various organs systems and in different diseases: skin diseases, coronary heart disease, obstructive lung disease and recurrent infection, and they reduce postoperative morbidity due to infection. Alpha-linolenic acid offers practical advantages in comparison with fish oil preparations. Unpleasant belching is reduced and only low levels of stabilisers (antioxidants) are needed in the formulation.

Key words: Polyunsaturated fatty acids (PUFAs), metabolism, nutrition, fish oil 
Die n-6- und n-3-PUFA sind essenzielle Bestandteile der Phospholipide in Zellmembranen. Einige n-3-PUFA, speziell die Eicosapentaensäure (EPA), sind auch Vorstufen von Eicosanoiden (u.a. Prostaglandine, Thromboxane und Leukotriene). Allerdings unterscheiden sich Eicosanoide, die aus der EPA entstehen, biologisch wesentlich von den Eicosanoiden aus Arachidonsäure, die zum Teil ausgesprochen negative Effekte haben, beispielsweise auf die Entstehung von Herz-Kreislauf-Erkrankungen. Es gibt zunehmend Hinweise darauf, dass n-3-PUFA Risikofaktoren für Herz-Kreislauf-Erkrankungen sowie für entzündliche und immuno-logische Krankheitsprozesse reduzieren oder hemmen können - dies aufgrund ihrer hemmenden Wirkung auf die Bildung von Arachidonsäure und der entsprechenden Eicosanoide.

Zwischen n-3- und n-6-PUFA besteht hinsichtlich der Prostaglandinbildung eine kompetitive Beziehung: Die Fettsäuren konkurrieren bei der Biosynthese der physiologisch wichtigen langkettigen und hochungesättigten Fettsäuren um das gleiche endoplasmatische Enzymsystem. Besonders „umkämpft“ ist die Delta-6-Desaturase; die Affinität zu diesem Enzym ist bei den n-3-Fettsäuren am höchsten und bei den n-9-Fettsäuren am geringsten. Weniger als $10 \%$ der zugeführten Linol- und Alpha-Linolensäure werden in die entsprechenden langkettigen Derivate umgesetzt. AlphaLinolensäure wird zu ca. 6\% zu EPA und $\mathrm{zu} 4 \% \mathrm{zu}$ Docosahexaensäure (DHA) metabolisiert [1]. In Abbildung 2 sind die bedeutendsten Metaboliten der PUFA dargestellt [2,3].

LA und ALA haben, gemessen an der Eicosanoidsynthese, eine gering andere biologische Wirksamkeit als ihre längerkettigen Derivate Arachidonsäure und EPA. Für eine einheitliche Bewertung der Bedarfsdeckung wäre also die Verwendung des Begriffs „n-6Fettsäuren-Äquivalent“ bzw. „n-3-Fettsäuren-Äquivalent" notwendig. Eine endgültige Definition solcher Äquivalente ist jedoch noch nicht möglich. Momentan ist der Erkenntnisstand wie folgt: In Experimenten von einer oder zwei Wochen Dauer war EPA je nach Ausgangssituation zwischen zwei- und

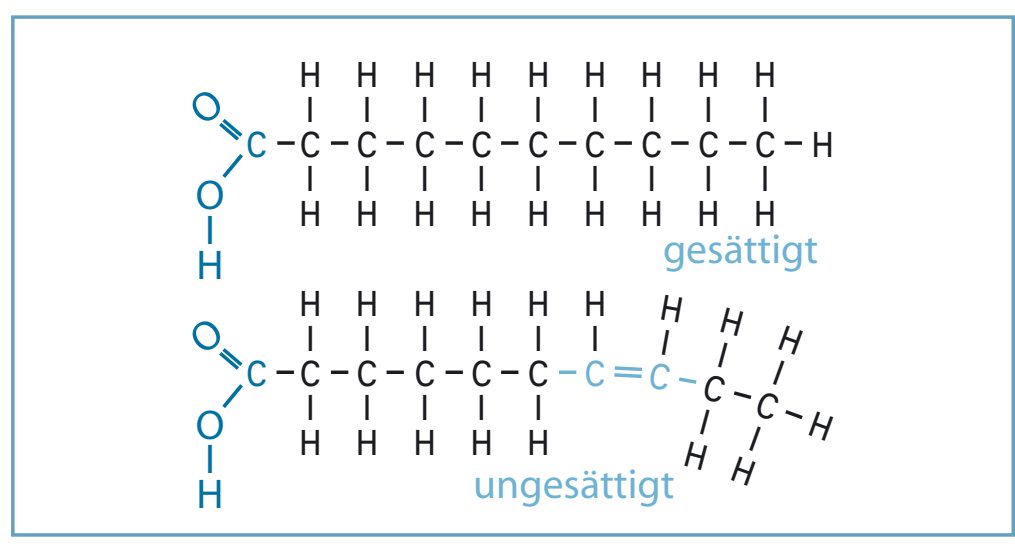

Abb. 1. Die chemischen Formeln von gesättigten und ungesättigten Fettsäuren.

zehnmal wirksamer als ALA [4]; diese Resultate müssten jedoch mit längeren Experimenten bestätigt werden. EPA und DHA werden auch in die Phospholipide der Zellmembranen verestert, was zum Beispiel die metabolische Aktivierung von Neutrophilen während der Phagozytose unspezifisch verändern kann [5].

Die Konversion von ALA zu EPA und DHA kann durch eine n-6-PUFA-reiche Diät um 40-50\% gehemmt werden. Die Proportion zwischen n-6- und n-3PUFA in der Ernährung sollte daher die Ratio 4 - 6 nicht überschreiten, denn die limitierte Konversionsrate kann in Anbetracht der spezifischen Funktionen von EPA und DHA kritisch sein/werden? Beim Erwachsenen bewirkt DHA eine Senkung der Triglyceridspiegel, einem bekannten kardiovaskulären Risikofaktor. In Zukunft wird der Einsatz einer adäquaten Dosis DHA notwendig sein, um eine genügende Versorgung mit diesem langkettigen Metaboliten sicherzustellen.

Die diversen PUFA sind in verschiedenen Ölen enthalten (Tabelle 1). Fünf bis sechs Fischmahlzeiten pro Woche (700 g) entsprechen ca. 7.5 g Fischöl täglich [6]. n-3-PUFA aus Fischöl müssen mit Antioxidantien wie Vitamin E kombiniert werden, damit sie stabilisiert werden und ihre Wirkung entfalten können [7].

\section{Mangelzustände}

Ein Mangel an essenziellen Fettsäuren ist sehr selten, da das Fettgewebe eines normalgewichtigen und vollwer- tig ernährten Erwachsenen über $500 \mathrm{~g}$ Linolsäure und $25 \mathrm{~g}$ Alpha-Linolensäure enthält. Ein Mangel an langkettigen n-6- bzw. n-3-Fettsäuren, speziell Arachidonsäure (n-6), EPA und DHA (n-3), kann während der Perinatalzeit vorkommen. Bei gesunden Erwachsenen ist ein solcher Mangel jedoch noch nie beobachtet worden.

Mit einem PUFA-Mangel ist allenfalls bei chronischer Fettmalabsorption oder bei fettfreier Ernährung zu rechnen, besonders bei fehl- und mangelernährten Personen (zum Beispiel infolge Magersucht). Die Wahrscheinlichkeit einer unzureichenden Versorgung mit essenziellen n-3- und n-6-Fettsäuren ist am grössten bei Menschen, deren Bedarf erhöht ist:

- Während der Wachstumsphasen (Frühgeborene, Teenager).

- Nach Stressphasen (Operationen, Verletzungen, Infektionen) [8].

- Bei Erkrankungen mit chronischen Verdauungs- oder Resorptionsstörungen; dies gilt insbesondere für Patienten nach grösseren Bauchoperationen oder nach schweren Unfällen.

- Eine ungenügende Einnahme von PUFA wurde bei älteren Patienten beschrieben [9].

- Bei Langzeittherapie mit Neuroleptika (Phenothiazine, Thioxanthene).

Bei Patientinnen mit Brustkrebs waren in einer Studie die Tumorgrösse und ein tiefer ALA-Gehalt im exzidierten Gewebe die zwei wichtigsten prognostischen Faktoren bezüglich Entwicklung von Metastasen [10]. Ob hier ein kausaler Zusammenhang besteht 
oder ob gar ein therapeutischer Ansatz entwickelt werden könnte, ist noch gänzlich offen. Verschiedene Autoren weisen auch hin auf ein erhöhtes Prostatakarzinomrisiko bei erhöhtem ALA-Konsum bzw. einer erhöhten Gewebekonzentration; die Datenlage ist jedoch widersprüchlich und es können diesbezüglich keine endgültigen Aussagen gemacht werden [11].

\section{Medizinische Anwendung der PUFA}

\section{PUFA in der Dermatologie}

Eine ungenügende Zufuhr von Linolsäure, der bedeutendsten n-6-PUFA in der normalen Epidermis, führt $\mathrm{zu}$ einer typischen Hautstörung mit Schuppenbildung und übermässigem Wasserverlust (Mangel an Sphingolipiden wie Acylceramid im Stratum corneum). Anders als im restlichen Organismus wird Linolsäure in der Haut durch die epidermische 15-Lipoxygenase hauptsächlich in 13-HydroxyOctadecadiensäure umgewandelt, die eine antiproliferative Wirkung im Gewebe hat. Im Vergleich zu Linolsäure spielt Arachidonsäure in der Haut eine eher untergeordnete Rolle; Arachidonsäure wird dort vorwiegend in 15-Hydroxy-Eicosatetraensäure (15-HETE) umgewandelt, die antiinflammatorische Eigenschaften aufweist [12].

Im Tierexperiment reduzieren ALA und Linolsäure die durch UV-Bestrahlung induzierte Hyperpigmentierung, da sich die Melaninsynthese reduziert und vermehrt Hornzellen abschilfern [13].

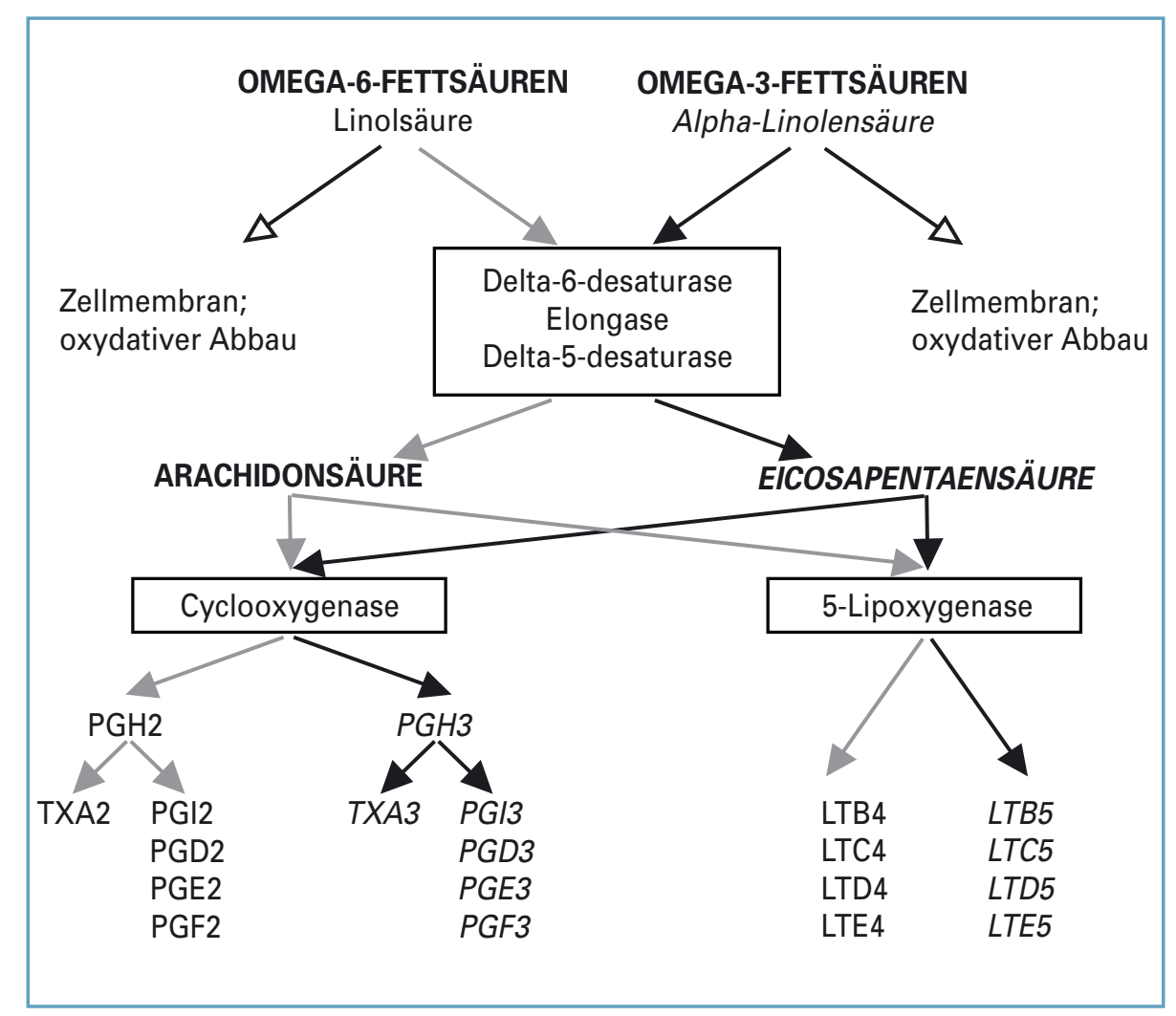

Abb. 2. PUFA und bedeutendste Metaboliten. Die kompetitive Hemmung des Arachidonsäureumbaus durch n-3-Fettsäuren wie Alpha-Linolensäure (sowie auch durch n-9-Fettsäuren) führt zu einer verminderten Synthese von Prostaglandinen der Serie 2 und von Leukotrienen der Serie 4 (Beteiligte Enzyme sind in $\square$ angegeben).
Bei Patienten mit Neigung zur Keloidbildung konnte im Vergleich zu gesunden Personen ein Zusammenhang mit ungenügender Zufuhr an ALA und anderen n-3-PUFA nachgewiesen werden. Die Fibroblasten der Dermis produzieren bei diesen Patienten ungehemmt Prostaglandine und freie Radikale mit der entsprechenden entzündlichen Kaskade und reaktiven Kolla- gensynthese [14,15]. Daher wird bei Patienten mit Neigung zur Keloidbildung eine Therapie (bzw. eine entsprechende Ernährungsweise) mit n-3PUFA, Vitamin E und Oligoelementen empfohlen; entsprechende klinische Studien stehen allerdings noch aus.

Bei Akne ist der Linolsäuregehalt im Sebum reduziert, trotzt normalen systemischen Werten. In einer Studie
Tab. 1. Quellen von n-3- und n-6-Fettsäuren

\section{n-3-Fettsäuren}

Alpha-Linolensäure (ALA)

Eicosapentaensäure (EPA) und Docosahexaensäure (DHA)

\section{n-6-Fettsäuren}

Linolsäure (LA)

Gamma-Linolensäure (GLA)

Arachidonsäure (AA)
Pflanzliche Öle, wie zum Beispiel Leinöl*), Rapsöl, Walnussöl und Sojaöl; grünes Blattgemüse

Fisch und Fischöl, besonders fette Seefische wie Lachs, Hering oder Makrele

Maiskeimöl, Färberdistelöl, Sonnenblumenöl; Margarinesorten, die als Hauptbestandteil pflanzliche Öle enthalten; Sojabohnen, Getreidearten

Samen von Nachtkerze, Borretsch und Schwarzen Johannisbeeren
In tierischen, fettreichen Produkten

* Enthält auch Lignan und PAF-Hemmer (PAF = Plättchen-aktivierender Faktor. Sehr oxidationsanfällig!) 
Tab. 2. Bedeutende Studien mit n-3-Fettsäuren, chronologisch geordnet

\begin{tabular}{|c|c|c|c|c|c|}
\hline $\begin{array}{l}\text { Autor, } \\
\text { Publikationsjahr }\end{array}$ & $\begin{array}{l}\text { Indikation und } \\
\text { Studiendesign }\end{array}$ & $\begin{array}{l}\text { Patien- } \\
\text { tenzahl }\end{array}$ & $\begin{array}{l}\text { Dosierung (p. Tag) } \\
\text { und Dauer der } \\
\text { Medikation }\end{array}$ & Zielvariablen & Ergebnisse \\
\hline $\begin{array}{l}\text { Kremer et al., } \\
1990[76]\end{array}$ & $\begin{array}{l}\text { - Rheumatoide } \\
\text { Arthritis } \\
\text { ndoppelblind }\end{array}$ & 54 & $\begin{array}{l}27 \mathrm{mg} / \mathrm{kg} \text { EPA + } \\
18 \mathrm{mg} / \mathrm{kg} \text { DHA vs. } \\
54 \mathrm{mg} / \mathrm{kg} \text { EPA + } \\
36 \mathrm{mg} / \mathrm{kg} \text { DHA vs. } \\
6.8 \mathrm{~g} \text { Ölsäure } \\
24 \text { Wochen }\end{array}$ & $\begin{array}{l}\text { LTB4 } \\
\text { Interleukin-1 beta } \\
\text { (IL-1 beta) } \\
\text { Ritchie's articular } \\
\text { index }\end{array}$ & $\begin{array}{l}\text { Gebesserte klinische Kriterien: } \\
\text { 5:45 mit Olivenöl, 8:45 mit der } \\
\text { tiefen EPA+DHA-Dosis und } \\
\text { 21:45 mit der hohen EPA+ } \\
\text { DHA-Dosis }\end{array}$ \\
\hline $\begin{array}{l}\text { Espersen et al., } \\
1992 \text { [77] }\end{array}$ & $\begin{array}{l}\text { Rheumatoide } \\
\text { Arthritis } \\
\text { - doppelblind }\end{array}$ & 32 & $\begin{array}{l}3.6 \mathrm{~g} \mathrm{n}-3 \text { Fisch- } \\
\text { Fettsäuren vs. } \\
\text { Placebo } \\
12 \text { Wochen }\end{array}$ & $\begin{array}{l}\text { (IL-1 beta) } \\
\text { TNF } \\
\text { Ritchie's articular } \\
\text { index }\end{array}$ & $\begin{array}{l}\text { Reduktion von (IL-1 beta), TNF } \\
\text { und Ritchie's articular index mit } \\
\text { Fettsäuren }\end{array}$ \\
\hline $\begin{array}{l}\text { Lau et al., } \\
1993 \text { [78] }\end{array}$ & $\begin{array}{l}\text { - Rheumatoide } \\
\text { Arthritis } \\
\text { - doppelblind }\end{array}$ & 64 & $\begin{array}{l}1.71 \mathrm{~g} \mathrm{EPA}+ \\
1.14 \mathrm{~g} \mathrm{DHA} \text { vs. } \\
\text { Placebo } \\
12 \text { Monate }\end{array}$ & $\begin{array}{l}\text { Verbrauch von } \\
\text { NSAR }\end{array}$ & $\begin{array}{l}\text { NSAR-Verbrauch reduziert um } \\
59 \% \text { vs. } 16 \%\end{array}$ \\
\hline $\begin{array}{l}\text { Geusens et al., } \\
1994[79]\end{array}$ & $\begin{array}{l}\text { - Rheumatoide } \\
\text { Arthritis } \\
\text { - doppelblind }\end{array}$ & 90 & $\begin{array}{l}2.6 \mathrm{~g} \text { n-3-Fettsäuren vs. } \\
1.3 \mathrm{~g} \text { n-3-Fettsäuren + } \\
3 \mathrm{~g} \text { Olivenöl vs. } \\
6 \mathrm{~g} \text { Olivenöl } \\
\text { ca. } 2 \text { Monate }\end{array}$ & $\begin{array}{l}\text { CGI } \\
\text { Gesamte Schmerz- } \\
\text { beurteilung } \\
\text { Reduktion von NSAR }\end{array}$ & $\begin{array}{l}\text { In den Zielkriterien war die } \\
\text { Dosierung von } 2.6 \mathrm{~g} \text { n-3-Fett- } \\
\text { säuren den anderen überlegen }\end{array}$ \\
\hline $\begin{array}{l}\text { Kremer et al., } \\
1995[80]\end{array}$ & $\begin{array}{l}\text { - Rheumatoide } \\
\text { Arthritis } \\
\text { - doppelblind }\end{array}$ & 66 & $\begin{array}{l}130 \text { mg/kg Omega-3- } \\
\text { Fettsäuren vs. } \\
9 \text { Kapseln Maisöl } \\
\text { Adjuvans zu } \\
\text { Diclofenac } \\
30 \text { Wochen }\end{array}$ & $\begin{array}{l}\text { Ritchie's articular } \\
\text { index } \\
\text { CGI } \\
\text { Morgensteife } \\
\text { IL-1 beta, IL-2, } \\
\text { IL-6, IL-8, TNF Alpha }\end{array}$ & $\begin{array}{l}\text { Signifikante Besserung } \\
\text { klinischer Variablen vs. } \\
\text { Maisöl; Reduktion von IL-1 beta }\end{array}$ \\
\hline $\begin{array}{l}\text { Nordstrom et al., } \\
1995 \text { [81] }\end{array}$ & $\begin{array}{l}\text { - Rheumatoide } \\
\text { Arthritis } \\
\text { n doppelblind }\end{array}$ & 22 & $\begin{array}{l}\text { n-3-Linolensäure vs. } \\
\text { Linolsäure } \\
\text { (Dosis unbekannt) } \\
3 \text { Monate }\end{array}$ & $\begin{array}{l}\text { Blutungszeit } \\
\text { CGI } \\
\text { funktioneller Status } \\
\text { Artikulationsscore } \\
\text { Index } \\
\text { Hämoglobin ESR } \\
\text { CRP }\end{array}$ & $\begin{array}{l}\text { Blutungszeit verlängert mit } \\
\text { n-3-Linolensäure, bei den rest- } \\
\text { lichen Variablen kein Unter- } \\
\text { schied }\end{array}$ \\
\hline $\begin{array}{l}\text { I) Morris et al., } \\
\text { 1995 [82] } \\
\text { II) Albert et al., } \\
1998 \text { [83] }\end{array}$ & $\begin{array}{l}\text { männliche Ärzte } \\
\text { in den USA } \\
\text { - Kohortenstudie }\end{array}$ & $\begin{array}{l}21 \\
185\end{array}$ & $\begin{array}{l}\text { Mahlzeiten mit Fisch } \\
\text { pro Woche } \\
\text { (F/W): }<1 \mathrm{~F} / \mathrm{W} ; 1 \mathrm{~F} / \mathrm{W} ; \\
\text { 2-4 F/W; > 5 F/W) } \\
\text { 4 Jahre }\end{array}$ & $\begin{array}{l}\text { I) } 281 \text { tödliche und } \\
\text { nicht-tödliche Herz- } \\
\text { infarkte (MI), } \\
173 \text { Schlaganfälle } \\
\text { und } 121 \text { kardio- } \\
\text { vaskuläre Todesfälle } \\
\text { II) Totale Mortalität } \\
\text { und Sekundenherztod } \\
\text { (SHT) }\end{array}$ & $\begin{array}{l}\text { I) RR } 1.0(95 \% \mathrm{Cl}), 1.1-2.3 \text { bei } \\
<1 \mathrm{~F} / \mathrm{W} ; 1.6,1.1-2.3 \text { bei } 1 \mathrm{~F} / \mathrm{W} \text {; } \\
1.4,1.0-2.0 \text { bei } 2-4 \mathrm{~F} / \mathrm{W} \text {; } \\
\text { 1.2, } 0.6-2.2 \text { bei }>5 \mathrm{~F} / \mathrm{W} \\
\text { II) RR } 0.48(95 \% \mathrm{Cl}) \text {, } \\
0.24-0.96 \text { bei > } 1 \mathrm{~F} / \mathrm{W}) \text {; } \\
\text { tiefere Gesamtmortalität }\end{array}$ \\
\hline $\begin{array}{l}\text { Simon et al., } \\
1995[84]\end{array}$ & $\begin{array}{l}\text { - Apoplex } \\
\text { - Fall-Kontroll- } \\
\text { Studie }\end{array}$ & 192 & $\begin{array}{l}\text { Blutspiegel verschiedener } \\
\text { Fettsäuren (stufenweise } \\
\text { Regressionsanalyse) }\end{array}$ & $\begin{array}{l}\text { Zunahme von } \\
\text { Phospholipid- } \\
\text { Stearinsäure resp. } \\
\text { Phospholipid- } \\
\text { Omega-3-Alpha- } \\
\text { Linolensäure }\end{array}$ & $\begin{array}{l}\Delta+1.37 \% \text { Stearinsäure entspre- } \\
\text { chen einer Erhöhung des Apo- } \\
\text { plexrisikos um } 37 \% \text {; } \\
\Delta+0.06 \% \text { Linolensäure } \\
\text { entsprechen einer Abnahme } \\
\text { des Apoplexrisikos um } 28 \%\end{array}$ \\
\hline $\begin{array}{l}\text { Ascherio et al., } \\
1996 \text { [85] }\end{array}$ & $\begin{array}{l}\text { Health } \\
\text { Professionals } \\
\text { - Kohortenstudie }\end{array}$ & $43^{\prime} 757$ & $\begin{array}{l}\text { Diät (u.a. mit } \\
\text { Alpha-Linolensäure) } \\
6 \text { Jahre }\end{array}$ & $\begin{array}{l}734 \text { Episoden von } \\
\text { koronarer Herz- } \\
\text { krankheit, hiervon } \\
505 \text { nicht-tödliche } \\
\text { Herzinfarkte und } \\
229 \text { Todesfälle }\end{array}$ & $\begin{array}{l}\text { Für Faserkonsum korrigierte, } \\
\text { signifikante Schutzwirkung von } \\
\text { Linolensäure }(\mathrm{RR}=0.41)\end{array}$ \\
\hline $\begin{array}{l}\text { Archer et al., } \\
1998[86]\end{array}$ & $\begin{array}{l}\text { Kohortenstudie } \\
\text { (Alter } \\
24-42 \mathrm{~J} \\
\text { Männer }+ \\
\text { Frauen) }\end{array}$ & $1 ` 672$ & Fischmahlzeiten & $\begin{array}{l}\text { Fibrinogen } \\
\text { Faktor VIII } \\
\text { von-Willebrand- } \\
\text { Faktor }\end{array}$ & $\begin{array}{l}\text { Kein Einfluss bei diätüblichen } \\
\text { Mengen }\end{array}$ \\
\hline $\begin{array}{l}\text { Stiefel et al., } \\
1999 \text { [87] }\end{array}$ & $\begin{array}{l}\text { - Diabetiker } \\
\text { randomisiert }\end{array}$ & 18 & $\begin{array}{l}330 \mathrm{mg} \text { DHA + } \\
630 \mathrm{mg} \text { EPA }\end{array}$ & $\begin{array}{l}\mathrm{HbA}(1 \mathrm{c}) \\
\text { Lipide und } \\
\text { Phospholipide der } \\
\text { Zellmembranen } \\
\text { neurale Leitung }\end{array}$ & $\begin{array}{l}\mathrm{HbA}(1 \mathrm{c})-2.00+1.9 \% \text { vs. } \\
-0.13+0.48 \% \\
\text { Signifikante Zunahme von C22:6 } \\
\text { (n-3) und n-3-Fettsäuren } \\
\text { in Zellmembranen } \\
\text { Signifikante Zunahme der } \\
\text { Reizleitungsgeschwindigkeit }\end{array}$ \\
\hline
\end{tabular}


Tab. 2. Bedeutende Studien mit n-3-Fettsäuren, chronologisch geordnet

\begin{tabular}{|c|c|c|c|c|c|}
\hline $\begin{array}{l}\text { Autor, } \\
\text { Publikationsjahr }\end{array}$ & $\begin{array}{l}\text { Indikation und } \\
\text { Studiendesign }\end{array}$ & $\begin{array}{l}\text { Patien- } \\
\text { tenzahl }\end{array}$ & $\begin{array}{l}\text { Dosierung (p. Tag) } \\
\text { und Dauer der } \\
\text { Medikation }\end{array}$ & Zielvariablen & Ergebnisse \\
\hline $\begin{array}{l}\text { Hu et al., } \\
1999[88]\end{array}$ & - Kohortenstudie $\frac{7}{\mathrm{~F}}$ & $\begin{array}{l}76^{\prime} 283 \\
\text { Frauen }\end{array}$ & $\begin{array}{l}\text { Gehalt an Linolensäure } \\
\text { in der Diät }\end{array}$ & $\begin{array}{l}\text { Tödliche und nicht- } \\
\text { tödliche Herzinfarkte } \\
\text { (MI) }\end{array}$ & $\begin{array}{l}\text { Tödliche } \mathrm{MI}=232 ; \\
\mathrm{RR}=0.55 ; 0.32-0.94(95 \% \mathrm{Cl}) \\
\text { Nicht-tödliche } \mathrm{MI}=597, \\
\mathrm{RR}=0.85 ; 0.61-1.19\end{array}$ \\
\hline $\begin{array}{l}\text { Gadek et al., } \\
1999 \text { [89] }\end{array}$ & $\begin{array}{l}\text { - Durch Sepsis } \\
\text { induziertes } \\
\text { Acute respiratory } \\
\text { distress syndrome } \\
\text { (ARDS) } \\
\text { - randomisiert }\end{array}$ & 146 & $\begin{array}{l}\text { EPA (Fischöl) + GLA vs. } \\
\text { isonitrogene, } \\
\text { isokalorische } \\
\text { Standarddiät }\end{array}$ & $\begin{array}{l}\text { a) Ventilatorische } \\
\text { Unterstützung } \\
\text { b) Tage in Intensiv- } \\
\text { pflege } \\
\text { c) Organversagen } \\
\text { d) Leukozyten in } \\
\text { Bronchoalveolärer } \\
\text { Spülung }\end{array}$ & $\begin{array}{l}\text { a) } 11 \text { vs. } 16.3 \text { Tage } \\
\text { b) } 12.8 \text { vs. } 17.5 \text { Tage } \\
\text { c) } 4 / 51 \text { vs. } 13 / 47 \text { Patienten } \\
\text { d) } 2.5 \text {-mal geringer }\end{array}$ \\
\hline $\begin{array}{l}\text { Goodfellow et al., } \\
2000 \text { [90] }\end{array}$ & $\begin{array}{l}\text { - Hyper- } \\
\text { cholesterinämie } \\
\text { doppelblind }\end{array}$ & 30 & $\begin{array}{l}4 \mathrm{~g} \mathrm{n} \text {-3-Fettsäuren vs. } \\
\text { Placebo } \\
4 \text { Monate }\end{array}$ & $\begin{array}{l}\text { Fluss-bedingte } \\
\text { arterielle Erweiterung } \\
\text { (FMD) } \\
\text { Triglyceride }\end{array}$ & $\begin{array}{l}\text { FMD }+120 \% \\
\text { Triglyceride von } 2.07 \mathrm{zu} \\
1.73 \mathrm{mmol} / \mathrm{l}\end{array}$ \\
\hline $\begin{array}{l}\text { Okamoto et al., } \\
2000 \text { [91] }\end{array}$ & Asthma & 7 und 7 & $\begin{array}{l}\text { Nachtkerzenöl } \\
\text { (n-3) vs. Maisöl (n-6) } \\
4 \text { Wochen }\end{array}$ & $\begin{array}{l}\text { Leukozytäre } \\
\text { Produktion von LTB4 } \\
\text { und LTC4 } \\
\text { PEF, FVC, FEV1, V(25) }\end{array}$ & $\begin{array}{l}\text { LTB4 und LTC4 signifikant } \\
\text { erhöht nach zwei Wochen } \\
\text { FVC und FEV1 zwischen } \\
\text { Gruppen }\end{array}$ \\
\hline
\end{tabular}

wurden durch die topische Applikation von Linolsäure die typischen Akne-Mikrokomedonen innerhalb eines Monats um ca. $25 \%$ reduziert [16]. Dementsprechend zeigten „in vitro“-Studien auch eine durch Linolsäure induzierte Hemmung der Phagozytose und der leukozytären Produktion von Peroxiden [17]; auch hier stehen entsprechende klinische Studien noch aus.

Die Wirkung von PUFAs bei atopischer Dermatitis ist nicht genügend belegt [18]. Bei Neugeborenen mit erhöhtem Atopierisiko soll die präventive Gabe von Gamma-Linolensäure zwar nicht das Risiko, aber den Schweregrad der atopischen Symptome mildern, wie in einer doppelblinden, plazebokontrollierten Studie gezeigt wurde [18a].

Bei Patienten mit Psoriasis haben n-3-Fettsäuren keine nachweisbare Wirkung [19], sie können aber als Adjuvans bei einer Retinoid-Behandlung die Nebenwirkungen wie Hypertriglyzeridämie positiv beeinflussen [20].

Bei Patienten mit Keratosis follicularis ist ein Defizit an Delta-6-Desaturase beschrieben worden. Gleichzeitig wurden erhöhte Linolsäure- und ALA-Blutspiegel gemessen, während die Konzentrationen der entsprechenden Delta-6-Desaturase-Metaboliten vermindert waren [21].

\section{Gerinnung und Thrombozytenaggregation}

Die Thrombozytenaggregation wird durch n-6-Ölsäure in hohen Dosen gesteigert, durch ALA und andere n-3Fettsäuren hingegen gehemmt [22,23]. ALA kann in genügender Dosierung die Blutungszeit verlängern, ohne die Gerinnungsfaktoren wie Fibrinogen, Faktor VIII oder von-Willebrand-Faktor zu modifizieren. n-3-Fettsäuren bewirken eine geringfügige Blutdrucksenkung um ca. 3-4 mmHg [24] und steigern gleichzeitig die arterielle Dehnbarkeit deutlich.

\section{PUFA-Supplemente \\ bei Neugeborenen}

Die Cochrane-Gruppe untersuchte randomisierte Studien, in denen ALA und Linolsäure als Supplemente in Säuglingsnahrung eingesetzt wurden. Als Schlussfolgerung ergab sich, dass die vorhandenen Daten keine Besserung der visuellen oder kognitiven Entwicklung der Säuglinge aufzeigen. Auch eine Wachstumsförderung lässt sich nicht feststellen [25,26]. Allerdings kann der DHA-Status der Neugeborenen durch die Verabreichung von ALA während der Schwangerschaft verbessert werden [27].

\section{Entzündungshemmung durch PUFA}

Verschiedene Studien beweisen die antiinflamatorische Wirkung von n-3Fettsäuren und deren klinischen Nutzen bei entzündlichen Prozessen [28,29]. Als Beispiel hier die Studie von WACHTLER et al. [30]: 40 Patienten erhielten nach grossen Baucheingriffen während fünf Tagen parenteral n-3-Fettsäuren. Anschliessend wurde ein leicht höherer Gehalt an leukozytären Leukotrienen B5 gemessen (nur geringfügig proinflammatorisch), aber auch eindeutig geringere Mengen an an Tumor Necrosis Factor (TNF)-Alpha festgestellt, im Vergleich zu den leukozytären Leukotrienen B4, Interleukin1 beta und Interleukin-6 und Interleukin-10.

Bei Patienten mit Dyslipidämie, die eine ALA-reiche Diät einhielten, wurde eine Senkung des C-reaktiven Proteins und von Interleukin-6 beschrieben [31]. Bei gesunden Probanden scheinen n3-Fettsäuren die Immunfunktionen aber nicht zu beeinflussen [32,33,34].

PUFA bei rheumatoider Polyarthritis Patienten mit rheumatoider Polyarthritis profitieren klinisch von der Einnahme von langkettigen n-3-Fettsäuren in Form von Supplementen, allerdings 
müssen diese Supplemente täglich als Zusatz zur Standardtherapie eingenommen werden. Bezüglich Wirksamkeit konnte zwischen ALA und Linolsäure (als Placebo) in einer Studie kein Unterschied gezeigt werden, was an der relativ kurzen Studiendauer liegen könnte [35].

\section{PUFA bei}

\section{gastrointestinalen Erkrankungen}

Bei Patienten mit Ulkus duodeni wurden tiefe Arachidonsäure-, ALA- und DHA-Spiegel beschrieben, die sich nach der Abheilung des Ulkus durch Protonenpumpenhemmer normalisierten [36].

Der therapeutische Einsatz von PUFA wird auch bei entzündlichen Darmerkrankungen wie Colitis ulcerosa und Morbus Crohn diskutiert [37]. In einer kleinen Doppelblindstudie mit Colitis-Patienten $(n=42)$ bewirkte Nachtkerzenöl einen Anstieg der erythrozytären Dihomogamma-Linolensäure (DGLA) und eine Besserung der Stuhlkonsistenz, nicht aber der anderen klinischen, endoskopischen oder histologischen Kriterien. Die Gabe von Fischöl war in dieser Studie unwirksam [38]. In einer anderen Studie wurde bei Colitis-Patienten in Remission die 5-ASA-Therapie abgesetzt und durch die tägliche Gabe von $5.1 \mathrm{~g}$ n-3PUFA bzw. Placebo ersetzt $(n=64)$; obwohl die Patienten der Verum-Gruppe signifikant länger in Remission blieben, war die Häufigkeit von Rezidiven nach zwei Jahren in Verum- und Placebo-Gruppe vergleichbar hoch [39].

Die epidemiologischen Daten sprechen dafür, dass die Ernährung und besonders die n-3-PUFA bei Morbus Crohn eine bedeutende Rolle spielen [40]. Trotzdem sind die Studienergebnisse einer PUFA-Therapie bei Morbus Crohn noch widersprüchlich $[41,42,43]$. Die Wirkung von n-3-PUFA kann wahrscheinlich durch die gleichzeitige Verabreichung von Antioxidantien verbessert werden [44].

\section{Die kardiovaskuläre}

\section{Schutzwirkung der PUFA}

Epidemiologische und prospektive Diät-Studien zeigen, dass ALA einen kardioprotektiven Effekt haben muss [45]. Marckmann und Gronbaek identifizierten elf Studien, die sich mit dem
Zusammenhang zwischen n-3-Fettsäuren resp. Fischkonsum und Herzleiden befassten [46]. Die studierten Kohorten umfassten 116'764 Probanden. Von den vier qualitativ als gut befundenen Studien wurden die zwei grösseren mit so genannten „low risk“Probanden durchgeführt $(\mathrm{n}=44$ ‘'895 und 20`051, Tabelle 2) und zeigten keinen messbaren Nutzen der fischreichen Kost. Bei den beiden anderen Studien waren die Probandenzahlen kleiner ( $\mathrm{n}=852$ und 1'822), die Studienteilnehmer wiesen jedoch ein höheres Risikoprofil auf; in diesen Studien konnte eine präventive Wirkung der fischreichen Kost auf das Risiko eines Todes durch koronare Herzkrankheit (KHK) beobachtet werden. Diese Studien suggerieren, dass 40-60 g Fisch pro Tag das KHK-Risiko um 40-60\% vermindern dürfte. Diese Befunde wurden durch eine neuere Meta-Analyse mit 19 Studien bestätigt [46a].

Eine italienische Studie konnte keinen Zusammenhang zwischen ALAKonsum und dem Risiko für einen nicht-tödlichen Myokardinfarkt feststellen [47]; eine Studie aus dem Jahr 2003 zeigt allerdings, dass bei Personen mit höheren ALA-Spiegeln im Fettgewebe das Risiko für einen nichttödlichen Herzinfarkt signifikant erniedrigt ist [48]. In der National Heart, Lung, and Blood Institute Family Heart Study ergab sich, dass bei Personen mit dem höchsten ALA-Konsum die Triglyzeridspiegel um durchschnittlich $12 \%$ niedriger waren als bei den Personen mit dem geringsten Konsum [49]. In derselben Studie konnte auch gezeigt werden, dass bei hohem ALA-Konsum ein geringeres Risiko für Plaques in den Karotiden besteht [50]. Bei Patienten mit Hypercholesterinämie verbesserte sich die endothelabhängige Vasodilatation durch die Gabe von ALA [51].

In einer weiteren Studie wurden Patienten nach Myokardinfarkt über fünf Jahre beobachtet; eine mit ALA angereicherte Diät war der „Prudent“Diät der American Heart Association eindeutig überlegen (nur 8 vs. 20 Todesfälle) [52]. In der Lyon Heart Study mit 600 Patienten nach Myokardinfarkt konnte mit der ALA-reichen Kretamediterranen Diät - im Vergleich zur genannten „Prudent-Diät“ - die gesamte und die kardiale Mortalität um $70 \%$ reduziert werden [53,54]. Die Fall-Kontroll-Studie von SIMON et al. (Tabelle 2) suggeriert ein geringeres, vom Raucherstatus und Blutdruck unabhängiges Hirnschlagrisiko bei höheren ALA-Spiegeln. Der Vollständigkeit halber sei erwähnt, dass bisher keine Wirkung der PUFA nach koronarer Angioplastik nachgewiesen wurde, trotz gross angelegter Kurzzeitstudien.

\section{PUFA-Wirkung bei Nephropathien?}

In einer Studie erhielten 42 Patienten mit IgA-Nephropathie täglich 6 g EPA und DHA oder Maisöl; es zeigte sich jedoch keine Besserung der nephrologischen Variablen [55]. Die Behandlung von je 25 Patienten nach Nierentransplantation mit täglich $6 \mathrm{~g}$ Fischöl bzw. Kokosnussöl bewirkte ebenfalls keine Besserung der Zielvariablen wie Anzahl Abstossreaktionen, glomeruläre Filtrationsrate, Blutdruck etc. [56]. Eine kleine Studie mit 9 Patienten, die an einer Lupusnephritis litten, zeigte mit täglich 30 g Leinöl (ALA) eine Abnahme der Kreatininspiegel und der Proteinurie [57]; eine weitere Studie an 26 Patienten mit Lupusnephritis, die mit n-3-Fischöl behandelt wurden, zeigte jedoch keinen klinischen Nutzen [58].

\section{PUFA in der Pulmologie}

In zwei Übersichtsarbeiten wird gefolgert, dass epidemiologische Daten eine schützende Rolle von n-3-Fettsäuren besonders bei chronischer Bronchitis und Emphysem nahe legen [59,60]. Auch bei Asthma sollen n-3-Fettsäuren, die mit Antioxidantien kombiniert werden, eine präventive Funktion ausüben [61]. VENUTA et al. berichten über eine doppelblinde Studie an 20 Kindern mit rezidivierenden respiratorischen Infekten [62]. Die Kinder erhielten pro Tag $596 \mathrm{mg}$ Linolsäure und $855 \mathrm{mg}$ ALA. Die Anzahl infektiöser Episoden, die Tage mit Fieber sowie die Schulabwesenheiten waren in den Behandlungsperioden signifikant tiefer. Eine kleine Studie mit AsthmaPatienten zeigt ebenfalls eine signifikante Besserung mit Nachtkerzenöl. Auch bei allergischem Asthma wurden erste positive Resultate mit n-3-Fett- 
säuren erzielt [63]. In einer grossen Studie mit 146 erwachsenen Patienten, die an septischem Atemnotsyndrom (ARDS) litten, konnte mit EPA und GLA ein eindeutig günstigerer Verlauf erzielt werden.

\section{PUFA zur Immunonutrition}

Ältere prospektive Studien zeigen, dass Immundiäten, die mit n-3-Fettsäuren angereichert werden, die postoperative infektiöse Morbidität um bis zu 75\% senken können [64]. Dieser Befund wurde für die abdominale Chirurgie und abdominale Traumen bestätigt $[65,66]$. Die Diät bewirkte tiefere Spiegel von proinflammatorischen Zytokinen und eine verzögerte Hypersensitivität bei 42 Patienten nach abdominalen Eingriffen aufgrund einer Krebserkrankung [67]. Eine Studie mit 64 Aidspatienten zeigte keinen Einfluss auf die Anzahl der CD4- und CD8Zellen, den „viral load“, den TumorNekrose-Faktor oder die Lebensqualität [68]. Eine andere Studie zeigte jedoch einen positiven Einfluss auf den Tumor-Nekrose-Faktor und das Körpergewicht [69]. In einer kleineren Studie mit Arginin, Spurenelementen und n3-Fettsäuren bei posttraumatischen Patienten konnte kein Vorteil gegenüber der Standarddiät nachgewiesen werden [70].

\section{PUFA in Psychiatrie und Neurologie}

In einer placebokontrollierten Studie über vier Monate zeigten Omega-3Fettsäuren bei 40 Patienten mit bipolaren Depressionen eine positive Wirkung. Ermutigende Daten gibt es auch für Schizophrenie-Patienten [71].

Die Deutsche Multiple Sklerose Gesellschaft hat Ernährungsempfehlungen für MS-Patienten herausgegeben; die Modifikation der Fettzufuhr steht im Vordergrund: Tierische und gesättigte Fette werden strikt begrenzt, stattdessen werden täglich vier bis zehn Teelöffel hochwertiges Öl empfohlen, dazu täglich ein Löffel Lebertran oder ein entsprechendes Präparat. Zudem wird zu einer vegetarisch betonten Ernährung mit vielen Ballaststoffen, viel Fisch und wenig zuckerhaltigen Lebensmitteln geraten [72].

\section{Schlussfolgerungen}

(in Anlehnung an Ref. 1 und 73)

- Im Sinne einer präventiv wirksamen Zusammensetzung der mehrfach ungesättigten Fettsäuren in der Nahrung von Gesunden sprechen sich die Deutsche, Österreichische und Schweizerische Gesellschaft für Ernährung dafür aus, dass das Verhältnis von Alpha-Linolensäure (n-3) zu Linolsäure (n-6) auf mindestens 1:5 erhöht wird.

- Die Wirkung von Alpha-Linolensäure auf die Triacylglycerolspiegel ist erwiesen, sie ist jedoch schwächer als die der entsprechenden Metaboliten bzw. von Fisch stammenden n-3Fettsäuren (EPA und DHA) [74].

- Die Nahrung sollte zum Schutz vor der Oxidation ungesättigter Fettsäuren wenigstens $0.4 \mathrm{mg}$ Tocopherol-Äquivalente pro Gramm Dienfettsäureäquivalent enthalten.

- Alpha-Linolensäure und Linolsäure spielen in der Haut eine bedeutende Rolle (Impermeabilisation der Hornschicht, Entzündungshemmung, antiproliferative Wirkung, Hemmung der UV-Wirkung).

- Der Konsum von Fisch reduziert wahrscheinlich die Inzidenz der KHK; daher sollte jeder mindestens eine Fischmahlzeit pro Woche einnehmen. Aktive Substanzen sind hier wahrscheinlich n-3-PUFA.

- Alpha-Linolensäure reduziert das KHK-Risiko um ca. 70\% [75]. Obwohl die Daten noch unvollständig sind, scheint die Einnahme von täglich 2 Gramm Alpha-Linolensäure bzw. einem Prozent der Energie angezeigt.

- Eine schützende Wirkung der n-3Fettsäuren bei rheumathoider Arthritis ist für hoch dosiertes Fischöl belegt; für Alpha-Linolensäure ist die Wirkung wahrscheinlich, der Nachweis steht jedoch noch aus.

- Eine schützende Wirkung der n-3Fettsäuren bei obstruktiven Lungenleiden und rezidivierenden Infekten scheint relativ gut belegt.

- Der Nutzen von n-3-PUFA bei inflammatorischen Darmleiden wie Colitis ulcerosa und Morbus Crohn ist noch nicht befriedigend geklärt.

- Eine Immunonutrition mit Arginin-,
RNS- und Omega-3-Fettsäuren-Supplementa zur Senkung der postoperativen infektiösen Morbidität dürfte ein etabliertes Verfahren sein; die spezifische Rolle der n-3-Fettsäuren lässt sich hier jedoch kaum isolieren.

- Alpha-Linolensäure bietet praktische Vorteile gegenüber Fischölpräparaten: kein unangenehmes Aufstossen für den Patienten und geringerer Bedarf an Stabilisatoren (Antioxidantien) in der galenischen Darreichungsform.

This paper is an updated summary of an expert-workshop sponsored by BIOMED AG, Dübendorf.

\section{Literatur}

1 Gerster $\mathrm{H}$ : Can adults adequately convert alpha-linolenic acid (18:3n-3) to eicosapentaenoic acid (20:5n-3) and docosahexaenoic acid (22:6n-3)? Int J Vitam Nutr Res (1998) 68(3) 159-73

2 Linder MC: Nutritional Biochemistry and Metabolism. Elsevier Ed., 1991, 59

3 Ziboh VA, Miller CC, Cho Y: Metabolism of polyunsaturated fatty acids by skin epiderma enzymes: generation of antiinflammatory and antiproliferative metabolites. Am J Clin Nutr 2000 Jan; 71(1 Suppl):361S-6S

4 DGE, OeGE, SGEF \& SGE Referenzwerte für die Nährstoffzufuhr, Verlag Deutsche Gesellschaft für Ernährung, 1. Auflage, 2000, 53-57

5 Magaro M, Zoli A, Altomonte L, Mirone L, De Sole P, Di Mario G, De Leo E: Effect of fish oil on neutrophil chemiluminescence induced by different stimuli in patients with rheumatoid arthritis. Ann Rheum Dis (1992 Jul) 51(7):877-80

6 Fahrer $\mathrm{H}$, Hoeflin $\mathrm{F}$, Lauterburg $\mathrm{BH}$, Peheim E, Levy A, Vischer TL: Diet and fatty acids: can fish substitute for fish oil? Clin Exp Rheumatol (1991 Jul-Aug) 9(4):403-6

7 Jokela R, Engstrom K, Wallin R, Saldeen T: Effect of in vitro stability of dietary fish oil on lipid peroxidation and prostanoids in vivo. Ups J Med Sci (1998) 103(3):213-21

8 Fischer S, Kissling W, Kuss HJ: Schizophrenic patients treated with high dose phenothiazine or thioxanthene become deficient in polyunsaturated fatty acids in their thrombocytes. Biochem Pharmacol 1992 Jul 22 44(2):317-23

9 Schmuck A, Villet A, Payen N, Alary J, Franco A, Roussel AM: Fatty acid nutriture in hospitalized elderly women. Am Coll Nutr 1998 Oct 17(5):448-53

10 Bougnoux $P$, Koscielny $S$, Chajes V, Descamps P, Couet C, Calais G: alpha-Linolenic acid content of adipose breast tissue: a host determinant of the risk of early metastasis in breast cancer. Br J Cancer (1994 Aug) 70(2):330-4

11 Attar-Bashi NM, Frauman AG, Sinclair AJ: Alpha-linolenic acid and the risk of prostate cancer. What is the evidence? J Urol (2004 Apr) 171(4):1402-7

12 Ziboh VA, Miller CC, Cho Y: Metabolism of polyunsaturated fatty acids by skin epiderma enzymes: generation of antiinflammatory and 
antiproliferative metabolites. In: Am J Clin Nutr (2000 Jan) 71(1 Suppl):361S-6S

13 Ando H, Ryu A, Hashimoto A, Oka M, Ichihashi M: Linoleic acid and alpha-linolenic acid lightens ultraviolet-induced hyper-pigmentation of the skin.Arch Dermatol Res (1998 Jul) 290(7):375-81

14 Louw L Dannhauser A Keloids in rural black South Africans. Part 2: dietary fatty acid intake and total phospholipid fatty acid profile in the blood of keloid patients. In: Prostaglandins Leukot Essent Fatty Acids (2000 Nov) 63(5):247-53

15 Louw L: Keloids in rural black South Africans. Part 3: a lipid model for the prevention and treatment of keloid formations. In: Prostaglandins Leukot Essent Fatty Acids (2000 Nov) 63(5):255-62

16 Letawe C, Boone M, Pierard GE: Digital image analysis of the effect of topically applied linoleic acid on acne microcomedones. Clin Exp Dermatol (1998 Mar) 23(2):56-8

17 Akamatsu H, Komura J, Miyachi Y, Asada Y, Niwa Y: Suppressive effects of linoleic acid on neutrophil oxygen metabolism and phagocytosis. J Invest Dermatol (1990 Sep) 95(3): 271-4

18 van Gool CJ, Zeegers MP, Thijs C: Oral essential fatty acid supplementation in atopic dermatitis - a meta-analysis of placebo-controlled trials. Br J Dermatol (2004 Apr) 150(4):728-40

18a van Gool CJ, Thijs C, Henquet CJ, van Houwelingen AC, Dagnelie PC, Schrander J, Menheere PP, van den Brandt PA: Gammalinolenic acid supplementation for prophylaxis of atopic dermatitis - a randomized controlled trial in infants at high familial risk. Am J Clin Nutr (2003 Apr) 77(4):943-51

19 Endres S, De Caterina R, Schmidt EB, Kristensen SD: n-3 polyunsaturated fatty acids: update 1995. Eur J Clin Invest (1995 Sep) 25(9):629-38

20 Frati C, Bevilacqua L, Apostolico V: Association of etretinate and fish oil in psoriasis therapy. Inhibition of hypertriglyceridemia resulting from retinoid therapy after fish oil supplementation. Acta Derm Venereol Suppl (Stockh) (1994) 186:151-3

21 Oxholm A, Oxholm P, da Cunha Bang F, Horrobin DF: Abnormal essential fatty acid metabolism in Darier's disease. Arch Dermatol (1990 Oct) 126(10):1308-11

22 Allman MA, Pena MM, Pang D: Supplementation with flaxseed oil versus sunflowerseed oil in healthy young men consuming a low fat diet: effects on platelet composition and function. Eur J Clin Nutr 1995 Mar; 49(3):169-78

23 Mutanen M, Freese R: Polyunsaturated fatty acids and platelet aggregation. Curr Opin Lipidol 1996 Feb; 7(1):14-9

24 Morris MC: Dietary fats and blood pressure. J Cardiovasc Risk (1994 Jun) 1(1):21-30

25 Simmer K, Patole S: Longchain polyunsaturated fatty acid supplementation in preterm infants. Cochrane Database Syst Rev (2004) (1):CD000375

26 de Groot RH, Adam J, Jolles J, Hornstra: Alpha-linolenic acid supplementation during human pregnancy does not effect cognitive functioning. Prostaglandins Leukot Essent Fatty Acids (2004 Jan) 70(1):41-7

27 de Groot $\mathrm{RH}$, Hornstra G, van Houwelingen AC, Roumen F: Effect of alpha-linolenic acid supplementation during pregnancy on maternal and neonatal polyunsaturated fatty acid status and pregnancy outcome. Am J Clin Nutr (2004 Feb) 79(2):251-60

28 Kremer JM: Effects of modulation of inflammatory and immune parameters in patients with rheumatic and inflammatory disease receiving dietary supplementation of $n-3$ and n-6 fatty acids. Lipids (1996 Mar) 31 Suppl: S243-7

29 James MJ, Gibson RA, Cleland LG: Dietary polyunsaturated fatty acids and inflammatory mediator production. Am J Clin Nutr (2000 Jan) 71(1 Suppl):343S-8S

30 Wachtler P, Konig W, Senkal M, Kemen M, Koller M: Influence of a total parenteral nutrition enriched with omega-3 fatty acids on leukotriene synthesis of peripheral leukocytes and systemic cytokine levels in patients with major surgery. J Trauma (1997 Feb) 42(2): 191-8

31 Rallidis LS, Paschos G, Liakos GK, Velissaridou AH, Anastasiadis G, Zampelas A: Dietary alpha-linolenic acid decreases C-reactive protein, serum amyloid A and interleukin-6 in dyslipidaemic patients. Atherosclerosis (2003 Apr) 167(2):237-42

32 Kew S, Banerjee T, Minihane AM, Finnegan YE, Muggli R, Albers R, Williams CM, Calder PC: Lack of effect of foods enriched with plant- or marine-derived n-3 fatty acids on human immune function. Am J Clin Nutr (2003 May) 77(5):1287-95

33 Miles EA, Banerjee T, Dooper MM, M'Rabet $L$, Graus YM, Calder PC: The influence of different combinations of gamma-linolenic acid, stearidonic acid and EPA on immune function in healthy young male subjects. $\mathrm{Br} \mathrm{J}$ Nutr (2004 Jun) 91(6):893-903

34 Wallace FA, Miles EA, Calder PC: Comparison of the effects of linseed oil and different doses of fish oil on mononuclear cell function in healthy human subjects. Br J Nutr (2003 May) 89(5):679-89

35 Rothman D, DeLuca P, Zurier RB: Botanical lipids: effects on inflammation, immune responses, and rheumatoid arthritis. Semin Arthritis Rheum (1995 Oct) 25(2):87-96

36 Manjari V: Das UN Oxidant stress, anti-oxidants, nitric oxide and essential fatty acids in peptic ulcer disease. Prostaglandins Leukot Essent Fatty Acids 1998 Dec; 59(6):401-6

37 Belluzzi A, Boschi S, Brignola C, Munarini A, Cariani G, Miglio F: Polyunsaturated fatty acids and inflammatory bowel disease. Am J Clin Nutr (2000 Jan) 71(1 Suppl):339S-42S

38 Greenfield SM, Green AT, Teare JP, Jenkins AP, Punchard NA, Ainley CC, Thompson RP: $A$ randomized controlled study of evening primrose oil and fish oil in ulcerative colitis. Aliment Pharmacol Ther (1993 Apr) $7(2): 159-66$

39 Loeschke K, Ueberschaer B, Pietsch A, Gruber E, Ewe K, Wiebecke B, Heldwein W, Lorenz R: n-3 fatty acids only delay early relapse of ulcerative colitis in remission. Dig Dis Sci (1996 Oct) 41(10):2087-94

40 Hunter JO: Nutritional factors in inflammatory bowel disease. Eur J Gastroenterol Hepatol (1998 Mar) 10(3):235-7

41 Belluzzi A, Brignola C, Campieri M, Camporesi EP, Gionchetti P et al.: Effects of new fish oil derivative on fatty acid phospholipid-membrane pattern in a group of Crohn's disease patients. Dig Dis Sci (1994 Dec) 39(12): 2589-94

42 Endres S, Lorenz R, Loeschke K: Lipid treatment of inflammatory bowel disease. Curr Opin Clin Nutr Metab Care (1999 Mar) 2(2):117-20

43 Lorenz-Meyer H, Bauer P, Nicolay C, Schulz B, Purrmann J, Fleig WE, Scheurlen C, Koop I, Pudel V, Carr L: Omega-3 fatty acids and low carbohydrate diet for maintenance of remission in Crohn's disease. A randomized controlled multicenter trial. Study Group Members (German Crohn's Disease Study Group). Scand J Gastroenterol (1996 Aug) 31(8):778-85
44 Geerling BJ, Badart-Smook A, van Deursen $C$, van Houwelingen AC, Russel MG et al. Nutritional supplementation with N-3 fatty acids and antioxidants in patients with Crohn's disease in remission: effects on antioxidant status and fatty acid profile. Inflamm Bowel Dis (2000 May) 6(2):77-84

45 de Lorgeril M, Salen P: Alpha-linolenic acid and coronary heart disease. Nutr Metab Cardiovasc Dis (2004 Jun) 14(3):162-9

46 Marckmann P Gronbaek M: Fish consumption and coronary heart disease mortality. A systematic review of prospective cohort studies. Eur J Clin Nutr (1999 Aug) 53(8): 585-90

46a Whelton SP, He J, Whelton PK, Muntner P Meta-analysis of observational studies on fish intake and coronary heart disease. Am J Cardiol (2004 May 1) 93(9):1119-23

47 Tavani A, Pelucchi C, Negri $E$, et al: n-3 polyunsaturated fatty acids and nonfatal acute myocardial infarction. Circulation. 2001; 104: 2269-2272

48 Baylin A, Kabagambe EK, Ascherio A, et al. Adipose tissue $\alpha$-linolenic acid and nonfatal acute myocardial infarction in Costa Rica. Circulation. 2003; 107:1586-1591.

49 Djousse L, Hunt SC, Arnett DK, Province MA Eckfeldt JH, Ellison RC: Dietary linolenic acid is inversely associated with plasma triacylglycerol: the National Heart, Lung, and Blood Institute Family Heart Study. Am J Clin Nut (2003 Dec) 78(6):1098-102

50 Djousse L, Folsom AR, Province MA, Hunt SC, Ellison RC: Dietary linolenic acid and carotid atherosclerosis: the National Heart, Lung, and Blood Institute Family Heart Study. Am J Clin Nutr (2003 Apr) 77(4):819-25

51 Ros E, Nunez I, Perez-Heras A, Serra M, Gilabert R, Casals E, Deulofeu R: A walnut diet improves endothelial function in hypercholesterolemic subjects: a randomized crossover trial. Circulation (2004 Apr 6) 109(13):1609-14

52 Hartman IS: alpha-Linolenic acid: a preventive in secondary coronary events? Nutr Rev 1995 Jul; 53(7):194-7

53 de Lorgeril M, Renaud S, Mamelle N, Salen P, Martin JL, Monjaud I, Guidollet J, Touboul P, Delaye J: Mediterranean alpha-linolenic acidrich diet in secondary prevention of coronary heart disease [see comments] [published erratum appears in Lancet 1995 Mar 18; 345 (8951):738] Lancet 1994 Jun 11; 343(8911): 1454-9

54 Renaud S, de Lorgeril M, Delaye J, Guidollet J, Jacquard F, Mamelle N, Martin JL, Monjaud I, Salen P, Toubol P: Cretan Mediterranean diet for prevention of coronary heart disease. Am J Clin Nutr 1995 Jun; 61(6 Suppl):1360S-1367S

55 Pettersson EE, Rekola S, Berglund L, Sundqvist KG, Angelin B, Diczfalusy U, Bjork hem I, Bergstrom J: Treatment of IgA nephropathy with omega-3-polyunsaturated fatty acids: a prospective, double-blind, random ized study. Clin Nephrol (1994 Apr) 41(4) 183-90

56 Kooijmans-Coutinho MF, Rischen-Vos J, Hermans J, Arndt JW, van der Woude FJ: Dietary fish oil in renal transplant recipients treated with cyclosporin-A: no beneficial effects shown. In: J Am Soc Nephrol (1996 Mar) 7(3):513-8

57 Clark WF, Parbtani A, Huff MW, Spanner E, de Salis H, Chin-Yee I, Philbrick DJ, Holub BJ: Flaxseed: a potential treatment for lupus nephritis. Kidney Int 1995 Aug; 48(2):475-80

58 Clark WF, Parbtani A: Omega-3 fatty acid supplementation in clinical and experimental lupus nephritis. Am J Kidney Dis 1994 May 23(5):644-7 
59 Smit HA, Grievink L, Tabak C: Dietary influences on chronic obstructive lung disease and asthma: a review of the epidemiological evidence. Proc Nutr Soc (1999 May) 58(2): 309-19

60 Schwartz J: Role of polyunsaturated fatty acids in lung disease. Am J Clin Nutr 2000 Jan; 71(1 Suppl):393S-6S

61 Peat JK: Prevention of asthma. Eur Respir J (1996 Jul) 9(7):1545-55

62 Venuta A, Spano C, Laudizi L, Bettelli F, Beverelli $A$, Turchetto $E$ : Essential fatty acids: the effects of dietary supplementation among children with recurrent respiratory infections. J Int Med Res 1996 Jul-Aug; 24(4):325-30

63 Villani F, Comazzi R, De Maria P, Galimberti M: Effect of dietary supplementation with polyunsaturated fatty acids on bronchial hyperreactivity in subjects with seasonal asthma. Respiration (1998) 65(4):265-9

64 Alexander JW: Immunoenhancement via enteral nutrition. Arch Surg (1993 Nov) 128(11):1242-5

65 Kudsk KA, Minard G, Croce MA, Brown RO, Lowrey TS, Pritchard FE, Dickerson RN, Fabian TC: A randomized trial of isonitrogenous enteral diets after severe trauma. An immune-enhancing diet reduces septic complications. Ann Surg (1996 Oct) 224(4):531-40; discussion 540-3

66 Senkal M, Zumtobel V, Bauer KH, Marpe B, Wolfram G, Frei A, Eickhoff U, Kemen $M$ Outcome and cost-effectiveness of perioperative enteral immunonutrition in patients undergoing elective upper gastrointestinal tract surgery: a prospective randomized study. Arch Surg (1999 Dec) 134(12):1309-16

67 Gianotti L, Braga M, Fortis C, Soldini L, Vignali $A$, Colombo S, Radaelli G, Di Carlo V: A prospective, randomized clinical trial on perioperative feeding with an arginine-, omega-3 fatty acid-, and RNA-enriched enteral diet: effect on host response and nutritional status. JPEN J Parenter Enteral Nutr (1999 NovDec) 23(6):314-20

68 Pichard C, Sudre P, Hirschel B, Willimann C, Karsegard L, Schneider H: Double-blind randomized trial of arginine and W3 fatty acid oral supplement in HIV infection.In: Int Conf AIDS (1996 Jul 7-12) 11(1):314 (abstract no. Tu.B.2286)

69 Suttmann U, Ockenga J, Schneider H, Selberg $O$, Schlesinger A, Gallati H, Wolfram G, Deicher $\mathrm{H}$, Muller MJ: Weight gain and increased concentrations of receptor proteins for tumor necrosis factor after patients with symptomatic HIV infection received fortified nutrition support. J Am Diet Assoc 1996 Jun; 96(6): 565-9

70 Mendez C, Jurkovich GJ, Garcia I, Davis D, Parker A, Maier RV: Effects of an immuneenhancing diet in critically injured patients. J Trauma (1997 May) 42(5):933-40; discussion 940-1

71 Ärzte Zeitung, Internet, Update: 22.12.2000

72 Ärzte Zeitung, Internet, Update 05.06.2000

73 de Deckere EA, Korver O, Verschuren PM Katan MB: Health aspects of fish and n-3 polyunsaturated fatty acids from plant and marine origin. Eur J Clin Nutr 1998 Oct 52(10):749-53

74 Harris WS: n-3 fatty acids and serum lipoproteins: human studies. Am J Clin Nutr 1997 May; 65(5 Suppl):1645S-1654S

75 Renaud SC: Dietary management of cardiovascular diseases. Prostaglandins Leukot Essent Fatty Acids 1997 Oct; 57(4-5):423-7

76 Kremer JM, Lawrence DA, Jubiz W, DiGiacomo R, Rynes R, Bartholomew LE, Sherman
M: Dietary fish oil and olive oil supplementation in patients with rheumatoid arthritis. Clinical and immunologic effects. Arthritis Rheum (1990 Jun) 33(6):810-20

77 Espersen GT, Grunnet N, Lervang $\mathrm{HH}$, Nielsen GL, Thomsen BS, Faarvang KL, Dyerberg J, Ernst E: Decreased interleukin-1 beta levels in plasma from rheumatoid arthritis patients after dietary supplementation with n3 polyunsaturated fatty acids. Clin Rheumatol (1992 Sep) 11(3):393-5

78 Lau CS, Morley KD, Belch JJ: Effects of fish oil supplementation on non-steroidal antiinflammatory drug requirement in patients with mild rheumatoid arthritis -a double-blind placebo controlled study. $\mathrm{Br} \mathrm{J}$ Rheumatol (1993 Nov) 32(11):982-9

79 Geusens P, Wouters C, Nijs J, Jiang Y, Dequeker J: Long-term effect of omega-3 fatty acid supplementation in active rheumatoid arthritis. A 12-month, double-blind, controlled study. Arthritis Rheum (1994 Jun) 37(6):824-9

80 Kremer JM, Lawrence DA, Petrillo GF, Litts LL, Mullaly PM, Rynes RI, Stocker RP, Parhami N, Greenstein NS, Fuchs BR et al.: Effects of high-dose fish oil on rheumatoid arthritis after stopping nonsteroidal antiinflammatory drugs. Clinical and immune correlates. Arthritis Rheum (1995 Aug) 38(8):1107-14

81 Nordstrom DC, Honkanen VE, Nasu Y, Antila E, Friman C, Konttinen YT: Alpha-linolenic acid in the treatment of rheumatoid arthritis. A double-blind, placebo-controlled and randomized study: flaxseed vs. safflower seed. Rheumatol Int 1995; 14(6):231-4

82 Morris MC, Manson JE, Rosner B, Buring JE, Willett WC, Hennekens CH: Fish consumption and cardiovascular disease in the physicians' health study: a prospective study. Am J Epidemiol (1995 Jul 15) 142(2):166-75

83 Albert $\mathrm{CM}$, Hennekens $\mathrm{CH}$, O’Donnell CJ, Ajani UA, Carey VJ, Willett WC, Ruskin JN, Manson JE: Fish consumption and risk of sudden cardiac death [see comments] In: JAMA (1998 Jan 7) 279(1):23-8

84 Simon JA, Fong J, Bernert JT Jr, Browner WS: Serum fatty acids and the risk of stroke. Stroke 1995 May; 26(5):778-82

85 Ascherio A, Rimm EB, Giovannucci EL, Spiegelman D, Stampfer M, Willett WC: Dietary fat and risk of coronary heart disease in men: cohort follow up study in the United States [see comments] BMJ (1996 Jul 13) 313(7049):84-90

86 Archer SL, Green D, Chamberlain M, Dyer AR, Liu K: Association of dietary fish and n-3 fatty acid intake with hemostatic factors in the coronary artery risk development in young adults (CARDIA) study. Arterioscler Thromb Vasc Biol (1998 Jul) 18(7):1119-23

87 Stiefel P, Ruiz-Gutierrez V, Gajon E, Acosta D, Garcia-Donas MA, Madrazo J, Villar J, Carneado J: Sodium transport kinetics, cell membrane lipid composition, neural conduction and metabolic control in type 1 diabetic patients. Changes after a low-dose n-3 fatty acid dietary intervention. Ann Nutr Metab (1999) 43(2): 113-20

88 Hu FB, Stampfer MJ, Manson JE, Rimm EB, Wolk A, Colditz GA, Hennekens $\mathrm{CH}$, Willett WC: Dietary intake of alpha-linolenic acid and risk of fatal ischemic heart disease among women [see comments] Am J Clin Nutr (1999 May) 69(5):890-7

89 Gadek JE, DeMichele SJ, Karlstad MD, Pacht ER, Donahoe M, Albertson TE, Van Hoozen C, Wennberg AK, Nelson JL, Noursalehi M: Effect of enteral feeding with eicosapentaenoic acid, gamma-linolenic acid, and antioxidants in patients with acute respiratory distress syndrome. Enteral Nutrition in ARDS
Study Group. Crit Care Med 1999 Aug; 27(8): 1409-20

90 Goodfellow J, Bellamy MF, Ramsey MW, Jones CJ, Lewis MJ: Dietary supplementation with marine omega-3 fatty acids improve systemic large artery endothelial function in subjects with hypercholesterolemia. J Am Coll Cardiol (2000 Feb) 35(2):265-70

91 Okamoto M, Mitsunobu F, Ashida K, Mifune T, Hosaki Y, Tsugeno H, Harada S, Tanizaki $Y$ : Effects of dietary supplementation with n-3 fatty acids compared with n-6 fatty acids on bronchial asthma. Intern Med 2000 Feb; 39(2):107-11

\section{Korrespondenzadresse:}

Prof. Dr. med. Reinhard Saller

UniversitätsSpital Zürich

Dep. für Innere Medizin

Institut für Naturheilkunde

Rämistrasse 100, CH-8091 Zürich

reinhard.saller@usz.ch 